\title{
Hemangioma retrocricoideo
}

\section{Postcricoid hemangioma}

Thomas Schmidt $\mathrm{P}^{1}$, Pablo Ortega $\mathrm{R}^{1}$.

\begin{abstract}
RESUMEN
Los hemangiomas ubicados en la región retrocricoidea son raros. Los pocos casos publicados presentan síntomas dependientes de su tamaño y corresponden principalmente a disfagia y estridor. Este reporte presenta la primera serie de casos publicados en Chile y una revisión de la literatura. Se trata de lactantes que consultaron por estridor, laringitis recurrentes, disfagia, apneas y convulsiones; dos manejados con éxito con cirugía con láser $\mathrm{CO}_{2}$ y uno solo con observación. Se discute la importancia de la nasofibroscopía flexible en el estudio del estridor y la disfagia en la población pediátrica, y la eficacia del láser $\mathrm{CO}_{2}$ como opción de manejo para esta patología.
\end{abstract}

Palabras clave: Hemangioma, retrocricoideo, estridor.

\begin{abstract}
Hemangiomas located in the postcricoid region is rare. The few published cases display dependent symptoms of their size and mainly correspond to dysphagia and stridor. This report presents the first series of cases published in Chile and a revision of literature. We presented cases of suckling babies who consulted by stridor, recurrent laryngitis, dysphagia, apneas and convulsions; 2 deal successfully with surgery with laser $\mathrm{CO}_{2}$ and one single one with observation. We discussed to the importance of nasofibroscopia flexible in the study of the stridor and dysphagia in the pediatric population, and the effectiveness of laser $\mathrm{CO}_{2}$ like option of treatment for this pathology.

Key words: Hemangioma, postcricoid, stridor.
\end{abstract}

\section{INTRODUCCIÓN}

Los hemangiomas juveniles o congénitos, son los tumores benignos más frecuentes en la población pediátrica y aproximadamente dos tercios ocurren en la cabeza y cuello ${ }^{1}$. La ubicación retrocricoidea es rara con escasos reportes en la literatura. Manifiestan síntomas según su tamaño y lo más frecuente es el estridor y la disfagia. Presentamos la primera serie de casos publicados en Chile y una revisión de la literatura sobre el tema.

\section{CASO CLÍNICO 1}

Lactante femenina consulta por primera vez a los 3 meses de edad, por historia de estridor desde los primeros días de vida. Inicialmente no se realiza

Médico Otorrinolaringólogo Hospital Guillermo Grant Benavente 
estudio. Regresa a la edad de 1 año 3 meses por persistencia del estridor, disfagia, apneas y crisis convulsivas refractarias al tratamiento anticonvulsivante. El estudio con nasofibroscopía flexible en la consulta revela un tumor retrocricoideo de color azul y rosado, que aparece con el llanto y desaparece al tragar. Durante el examen al aumentar de tamaño provoca obstrucción del tracto aerodigestivo superior (Figura 1). Se realiza resonancia nuclear magnética (RM) con gadolinio, que evidencia imagen retrocricoidea hiperintensa en T1 (Figura 2). Se plantea el diagnóstico de hemangioma retrocricoideo y se realiza resección de la lesión con láser $\mathrm{CO}_{2}$. La nasofibroscopía posoperatoria comprueba la desaparición de la lesión (Figura 3) y la paciente evoluciona sin estridor ni hemorragia. La biopsia de la pieza quirúrgica confirma el diagnóstico de hemangioma. Hasta la fecha completa 1 año 10 meses de seguimiento, con abolición completa de los síntomas después de la cirugía, incluidas las convulsiones.

\section{CASO CLÍNICO 2}

Lactante masculino que consulta por estridor con el llanto y el esfuerzo desde aproximadamente los 8 meses de vida. Evaluado por otorrinolaringólogo (ORL) con nasofibroscopía flexible al año de edad se pesquisa un tumor retrocricoideo de aspecto rosado-violáceo que aumenta de tamaño al llorar y con el esfuerzo. Posteriormente se realiza evaluación de vía aérea con anestesia general no encontrando signos de la lesión en reposo. La lesión se hace evidente sólo al iniciar ventilación espontánea y esfuerzo respiratorio. Se realiza RM con gadolinio que no logra demostrar en forma clara la lesión. Es derivado al Hospital Guillermo Grant Benavente realizándose el diagnóstico de hemangioma retrocricoideo y por la progresión de los síntomas se decide la cirugía con láser $\mathrm{CO}_{2}$ que se realiza a los 17 meses de vida sin incidentes intraoperatorios y sin necesidad de realizar traqueostomía. Evoluciona bien en el posoperatorio con resolución completa del estridor. La biopsia de la pieza quirúrgica confirma el diagnóstico de hemangioma. Actualmente con un seguimiento de 1 año 4 meses se encuentra asintomático y sin evidencia de recidiva.

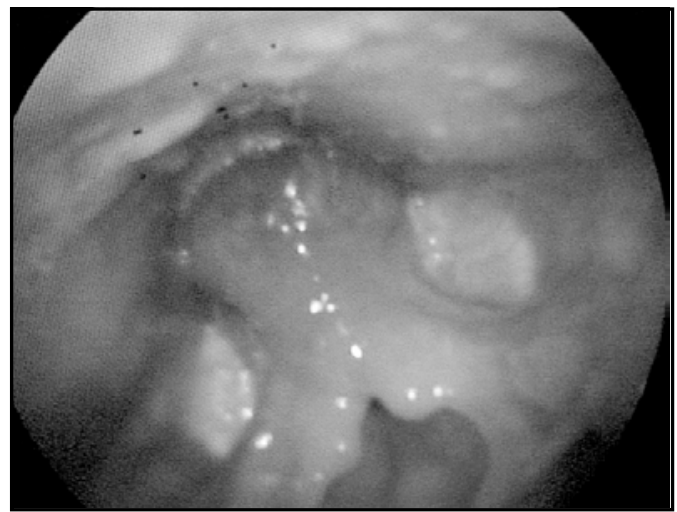

Figura 1. Hemangioma retrocricoideo a la nasofibroscopía flexible. Caso 1.

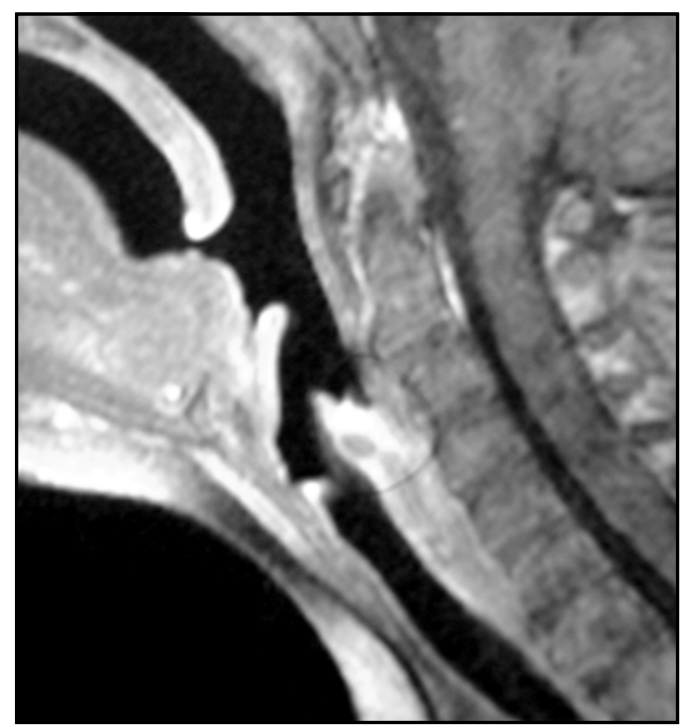

Figura 2. Resonancia nuclear magnética $\mathrm{T} 1$ con gadolinio. Caso 1.

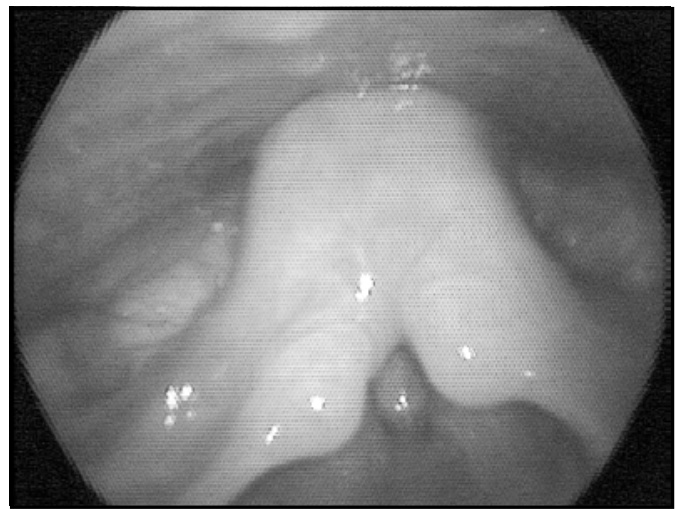

Figura 3. Nasofibroscopía control en posoperatorio. Caso 1. 


\section{CASO CLÍNICO 3}

Lactante masculino de 1 año 7 meses de edad es derivado a ORL por historia de laringitis agudas a repetición, con más de 10 episodios diagnosticados y manejados en la atención primaria durante su primer año de vida. No presentaba estridor de reposo, disfagia ni retraso del desarrollo pondoestatural. Al momento de la consulta se completan 6 meses sin nuevos episodios de laringitis agudas y el paciente se encuentra asintomático. Se realiza estudio con nasofibroscopía flexible y durante el llanto se evidencia un tumor retrocricoideo rosado-azul que aumenta de tamaño con el esfuerzo y el valsalva sin comprometer en ningún momento la vía aérea encontrándose limitado al área retrocricoidea (Figura 4). Se decide realizar un seguimiento cada 2 meses de la masa con nasofibroscopía flexible, a la fecha completa 1 año 2 meses de seguimiento asintomático y sin nuevas laringitis agudas obstructivas.

\section{DISCUSIÓN}

Los hemangiomas juveniles son los tumores benignos más frecuentes de la población pediátrica. Afectan entre el $1 \%-10 \%$ de los lactantes y son más frecuentes en prematuros, sexo femenino y raza caucásica ${ }^{1}$. La gran mayoría ocurren en la cabeza y el cuello, presentando una evolución típica caracterizada por una fase de proliferación durante los primeros 12 meses de vida y una progresiva involución posterior, que se completa aproximadamente en $50 \%$ a los 5 años y en $70 \%$ a los 7 años de edad ${ }^{1,2}$.

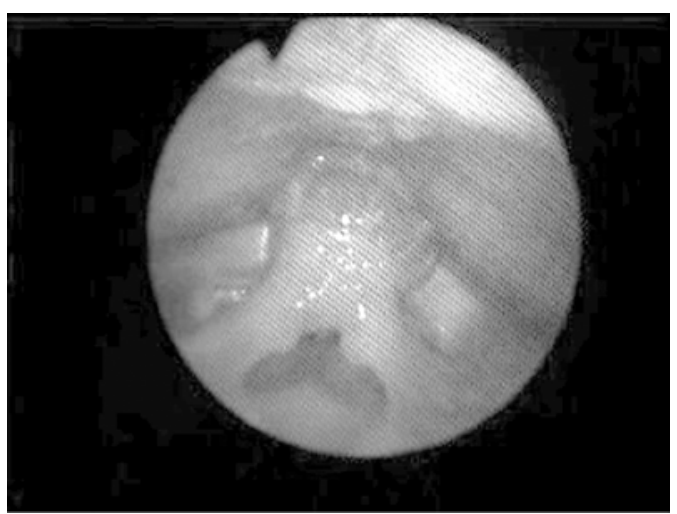

Figura 4. Nasofibroscopía flexible caso 3.
En el tracto aerodigestivo superior la ubicación más frecuente es la subglótica. Estas lesiones representan cerca del $1,5 \%$ de las alteraciones laríngeas congénitas, comprometen directamente la vía aérea y sin manejo alcanzan hasta $50 \%$ de mortalidad $^{2}$. La ubicación hipofaríngea es mucho más rara y los síntomas dependen de la ubicación y el tamaño de la lesión; incluyen disfagia, estridor, retraso del desarrollo y hematemesis ${ }^{1-3}$.

El hemangioma retrocricoideo juvenil fue descrito en 1987 por Goldsmith ${ }^{1}$, en un niño de 16 meses de edad que presentaba retraso del desarrollo e infecciones respiratorias a repetición. Desde esa fecha, los pocos reportes de casos clínicos publicados han permitido conocer el cuadro clínico, evolución y opciones de manejo.

El hemangioma retrocricoideo juvenil se presenta en la mayoría de los casos antes de los 2 años de edad ${ }^{4-6}$. Los síntomas dependen del tamaño de la lesión y pueden incluir estridor, disfagia, retraso del desarrollo, infecciones respiratorias a repetición y apneas ${ }^{5-8}$. No se han descrito hemangiomas cutáneos simultáneos. Las lesiones más pequeñas pueden ser completamente asintomáticas y ser hallazgos a la nasofibroscopía flexible $^{4}$, lo que ha permitido suponer que esta lesión, es más frecuente de lo que se cree.

El examen clave para el diagnóstico es la nasofibroscopía flexible ${ }^{3-8}$ en el paciente despierto. Permite visualizar un tumor retrocricoideo azul rosada que aumenta de tamaño con el llanto y el esfuerzo (Figura 1). La biopsia está contraindicada por el riesgo de hemorragia ${ }^{8}$. Otros estudios como el ecodoppler y la videofluoroscopía no permiten pesquisar con eficacia la lesión ${ }^{3-5}$. La evaluación de vía aérea con anestesia general puede no demostrar la lesión debido a que en reposo disminuye en forma notable su tamaño $0^{3,4}$. Por último la resonancia nuclear magnética puede demostrar una zona de hiperintensidad en T2 y en T1 posinyección de gadolinio:

En lo que respecta al manejo, depende de la presencia y severidad de los síntomas. En la serie de Awwad $^{8}$ se presentan 4 casos hallazgos a la nasofibroscopía flexible, sin síntomas atribuibles a la lesión, que fueron sometidos sólo a seguimiento semestral, con un máximo de 6 años revelando una involución progresiva de la lesión, similar al comportamiento de otros hemangiomas. 
Discolo publica en 2004, 4 casos con síntomas leves de disfagia que fueron manejados sólo con modificaciones de la dieta y de la alimentación ${ }_{5}$.

Cuando los síntomas son más severos las opciones incluyen los corticoesteroides, la cirugía láser y la cirugía abierta. Gauthier maneja exitosamente 2 casos con corticoesteroides sistémicos logrando involución de la lesión y desaparición de los síntomas ${ }^{6}$. Pero otros autores desestiman los corticoesteroides sistémicos por el riesgo de efectos adversos que impiden tratamientos de larga duración. Es así que también se ha usado inyecciones intralesionales con éxito en algunos casos ${ }^{8}$.

El láser $\mathrm{CO}_{2}$ y el potasio titanio-fosfato (KTP) se han usado también con éxito para tratar este tipo de lesiones, sin complicaciones ni recidivas descritas en las series publicadas ${ }^{3,7}$. Del mismo modo la cirugía abierta con abordaje a través de faringotomía lateral se ha usado para manejar casos severos que no responden a tratamiento médico con corticoesteroides ${ }^{8}$.

A la fecha no hay reportes sobre manejo de estos hemangiomas con propanolol sistémico, terapia que ha demostrado éxito en el manejo de hemangiomas subglóticos en el lactante ${ }^{9}$, por 10 que podría ser útil también en los hemangiomas retrocricoideos.

\section{CONCLUSIÓN}

Los hemangiomas retrocricoideos juveniles son tumores poco frecuentes, que deben incluirse en el diagnóstico diferencial del estridor y la disfagia del lactante. Esencial para su diagnóstico es la nasofibroscopía flexible con el paciente despierto. El manejo depende de la presencia y severidad de los síntomas, e incluye modificaciones en la alimentación, corticoesteroides, cirugía láser y cirugía abierta. La observación de algunos casos ha demostrado involución progresiva con los años, similar al comportamiento de los hemangiomas juveniles.

\section{BIBLIOGRAFÍA}

1. MacArthuR C. Head and neck hemangiomas of infancy. Curr Opin Otolaryngol Head Neck Surg 2006; 14(6): 397-405.

2. Pransky S, Canto C. Management of subglottic hemangioma. Curr Opin Otolaryngol Head Neck Surg 2004; 12(6): 509-12.

3. Goldsmith M, Strope G, Postma D, et al. Presentation and management of postcricoid hemangiomata in infancy. Laryngoscope 1987; 97 (7 Pt 1): 851-3.

4. Awwad R, Mortelli A. Postcricoid hemangioma of childhood: report of four cases. Ann Otol Rhinol Laryngol 2006; 115(3): 191-4.

5. Discolo C, Koltai P. Postcricoid hemangioma presenting as dysphagia a report of four cases. Arch Otolaryngol Head Neck Surg 2004; 130(12): 1420-2.

6. Gauthier R, El Makhloufi K, Geneviere F, et al. Postcricoid hemangioma: An overlooked cause of dysphagia in infants? - A case report. Dysphagia 2004; 19(1): 48-51.

7. ZUR K, WoOd R, ELLURU R. Pediatric postcricoid vascular malformation: A diagnostic and treatment challenge. Int J Pediatr Otorhinolaryngol 2005; 69(12): 1697-701.

8. Folia M, Naiman N, Dubois R, et al. Management of postcricoid and upper esophageal hemangioma. Int $\mathrm{J}$ Pediatr Otorhinolaryngol 2007; 71(1): 147-51.

9. Jephson C, Manunza F, et al. Successful treatment of isolated subglottic haemangioma with propranolol alone. Int $J$ Pediatr Otorhinolaryngol 2009; 73(12): 1821-3. 